\title{
A risk ranking strategy for network level bridge management
}

\author{
S. Sathananthan ; M.I. Rafiq \\ Faculty of Engineering and Physical Sciences, University of Surrey, Guildford, UK \\ T. Onoufriou \\ Department of Civil Engineering, Cyprus University of Technology, Cyprus
}

\begin{abstract}
At present almost all bridge owners and managers, i.e. Network Rail, Highways Agency and local authorities in the UK, carryout bridge inspections at regular intervals to collect information on condition and performance of bridges. Introduction of risk based approaches in the selection of inspection regimes can provide consistent safety levels within the network in a cost effective manner. This paper presents the development of a qualitative risk ranking strategy to characterize a network of bridges into groups with similar risk levels, which can form the basis for developing a risk based inspection regime over the network. There are a multitude of factors that affect risk. These factors are identified and rationally combined to present various attributes of bridges. A qualitative scoring system is then introduced which utilizes the attributes to rank bridges in terms of their relative risk. Sensitivity analysis is performed to quantify the effect of relative weights of the attributes on the risk scores. The methodology is demonstrated through its application on UK's Network Rail bridge stock comprising of about 40,000 bridges. The criteria to classify the severity of the attributes are established for the network. A random sample of bridges is ranked to illustrate the proposed methodology.
\end{abstract}

\section{INTRODUCTION}

Bridges deteriorate with time and need intermittent inspections to ensure that they are capable of carrying traffic. However, inspecting a network of bridges at regular intervals without considering issues related with bridge characteristics, functionality and significance may not provide optimum level of safety. A risk based inspection methodology can be useful in this context as it has the potential of rationalising the inspection planning with significant safety and cost benefits.

The early use of risk based inspections can be found in various industries such as air craft (e.g. Yang and Tang, 1974), and offshore (e.g. Faber et al, 1996, Onoufriou, 1999). These techniques have recently been utilised in transport infrastructure, in particular for maintenance management of bridges. [e.g. Sommer et al (1993), Frangopol et el, 1997, Liu \& Frangopol (2005)].

The previous studies have concentrated at an element/structure level or a small group of structures with similar characteristics, which are not readily applicable to a large stock of bridges. In this case, bridge specific analyses may not be feasible. Even if all the bridges are analysed individually and inspections are scheduled accordingly that will result in large variations in inspection intervals over the net- work causing practical difficulties and additional expenses. Instead of having the same inspection regime throughout the entire network or bridge specific inspection intervals, scheduling inspections for groups of bridges according to their relative risk would optimize the inspection resources in a cost effective manner. Therefore, it is necessary to categorise the bridges with similar characteristics into groups before developing a risk based inspection (RBI) methodology.

This paper presents a systematic risk ranking approach to a network of bridges considering the factors which influence their relative risk. This approach is beneficial to bridge owners to identify the safety critical bridges in their network and to plan interventions/inspections according to their risk levels to maintain the same risk level throughout the network.

\section{FACTORS AFFECTING RISK AND BRIDGE ATTRIBUTES}

Based on a study of available literature, and discussions with bridge owners, sixteen key factors have been identified as having a significant impact in determining the relative risk levels of bridges in a network. Many of the attributes affect the same attrib- 
ute of a bridge and hence can be grouped together. Five bridge attributes have been identified that are affected by these factors. A list of the key factors and their relationship with the bridge attributes is outlined in Figure 1. The attributes are elaborated in more detail in the following sub sections.

\subsection{Type}

In a network, there may be different forms of bridges with variations in the material type, span, etc. Since different materials have different mechanisms and rates of deterioration, material variation is one of the most significant factors affecting risk. Bridge age is another decisive factor. The rate of deterioration and hence the risk increases with age. Normally, a correlation between the bridge age and bridge form within a network can be observed. This may be due to the fact that the construction methods are similar over a certain period of time until another method is introduced. For instance, all the arch bridges owned by Network Rail were built more than 100 years ago (Bell, 2004). Therefore, these factors are grouped under a bridge attribute called 'Type'. This attribute will be used to divide a network into groups of bridges having similar characteristics in relation to risk.

\subsection{Environment}

The environment attribute is represented by the factors that are external to the structure but play an important role in determining the risk associated. These include loading, climate, location of a bridge and surroundings of the foundation. The effects of these factors will be different for different material types. For example, a reinforced concrete or a steel bridge located in a costal area may have severe corrosion attack, whereas a masonry bridge in a similar location might be least affected. Therefore, the environment attribute must be classified independently for each group of bridges defined through type attribute.

\subsection{Consequence}

The consequence attribute covers a wide range of factors from direct consequences of failure such as human loss, repair/replacement cost and the loss of income to indirect consequences such as traffic delay costs, environmental impacts, political impact, etc. Evaluating and interpreting all these consequences in numerical terms is difficult. Therefore, by considering parameters such as traffic flow and duration/cost of remedial works reasonable judgement can be made regarding the consequence. Higher traffic flow can be used as an indicator for high traffic delay cost, if flow is interrupted or high human loss in case of an accident. Similarly, length of a bridge (multiple spans or long span) may be re- lated to high maintenance costs and/or long duration of remedial works, etc.

\subsection{Inspectability}

If all main elements of a bridge are not inspectable then some defects may remain undetected, and result in higher risk of failure. The two scenarios leading to this include bridges having hidden details and bridges having difficulty in accessing main elements for inspection. For example, tenanted arches may have unidentified defects and if some parts of a bridge are under water then it may be difficult to inspect them during the visual inspection. These factors are grouped separately under the inspectability attribute.

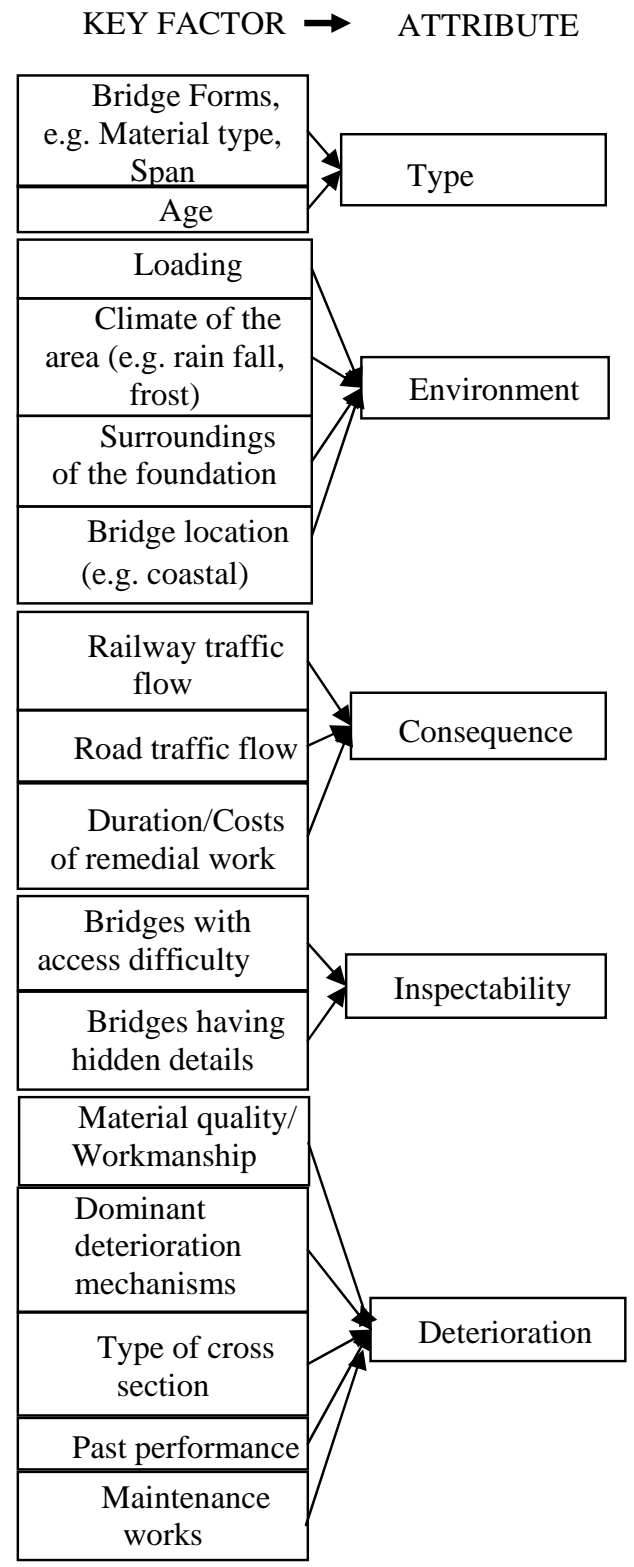

Figure 1. Relationship between key factors and bridge attributes 


\subsection{Deterioration}

The deterioration attribute incorporates all the factors that contribute to degradation of the structure with time. These include factors such as dominant deterioration mechanisms, workmanship and material quality, past performance and maintenance and the cross sectional make up of the structure. The dominant deterioration mechanisms will be different for each bridge type (as defined in section 2.1). Information on the other factors will also help in the classification, e.g. good workmanship and material quality and good past performance can be used as an indicator for a lower deterioration rate.

The bridge attributes in Sections 2.1 to 2.4 are all time independent and are utilised to provide an initial screening of the bridges in terms of their relative risk. The deterioration attribute provides the variation of the risk with time. This can be used, along with the initial relative risk, to develop a framework for risk based inspection planning of bridge stock.

\section{QUALITATIVE RISK SCORING SYSTEM}

A simple and practical approach for ranking the bridges is introduced by defining groups and sub groups of bridges in a network. 'Type' attribute is used as the basis to define the main groups. Three other attributes environment, consequence and inspectability are classified into two categories, in terms of the severity and subgroups are derived according to these classifications. Therefore, the subgroups serve as a risk ranking tool within a main group of bridges. A flow chart highlighting the grouping procedure is shown in Figure 2. A scoring system is then introduced to express the relative risk numerically.

\subsection{Relative risk score}

Initially, a score of 1 for the best cases and 2 for the worse cases are assigned to the attributes. For example, a score of 1 is allocated for the mild environment whereas 2 is assigned to the severe environment. Risk is defined as the multiplication of the probability of failure and consequence of failure. The environment and inspectability attributes are related to the probability of failure $\left(\mathrm{P}_{\mathrm{f}}\right)$ and the consequence attribute represents the consequence of failure $\left(\mathrm{C}_{\mathrm{f}}\right)$. Therefore, a score representing the relative risk of a subgroup, $\mathrm{R}$, can be expressed through Equation 1.

$$
\mathrm{R}=\left(\mathrm{W}_{1} \mathrm{~S}_{\mathrm{E}}+\mathrm{W}_{2} \mathrm{~S}_{\mathrm{I}}\right) \times \mathrm{S}_{\mathrm{C}}
$$

where $\mathrm{S}_{\mathrm{C}}=$ consequence score; $\mathrm{S}_{\mathrm{E}}=$ environment score; $\mathrm{S}_{\mathrm{I}}=$ inspectability score $; \mathrm{W}_{1}, \mathrm{~W}_{2}=$ Weight factors representing relative importance of $S_{E}$ and $S_{I}$ within the overall risk score $\mathrm{R}$.

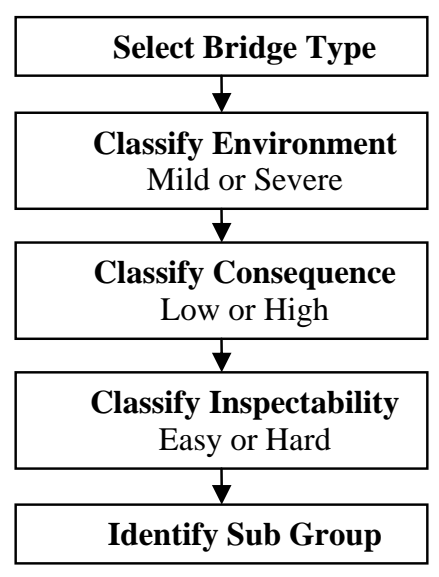

Figure 2. Selection procedure for grouping

\subsection{Weight factors for environment and inspectability scores}

The weighting of consequence attribute will not have any impact on the final risk score since it will be multiplied to the scores and $\mathrm{R}$ only represents the relative risk among the sub groups. Hence the relative weighting between environment and inspectability scores is considered in Equation 1. In some structures environment may be a critical factor in defining the relative risk whereas inspectability may be more critical in others. Sensitivity analysis is carried out to find the effect of weight factors $W_{1}$ and $\mathrm{W}_{2}$ on the final risk score, $\mathrm{R}$. The results are presented in Figure.3. Final risk scores can be arranged to vary between 1 and 2 by linear interpolation in order to compare the relative scores for different combinations of weight factors.

Initially, the risk score (R) of each sub-group for different combinations of environment $(\mathrm{E})$, inspectability (I) and consequence (C) factors (e.g. mild environment, hard inspectability and high consequence), while changing the relative weight of $\mathrm{W}_{1} / \mathrm{W}_{2}$ are calculated. These are plotted in Figure 3.

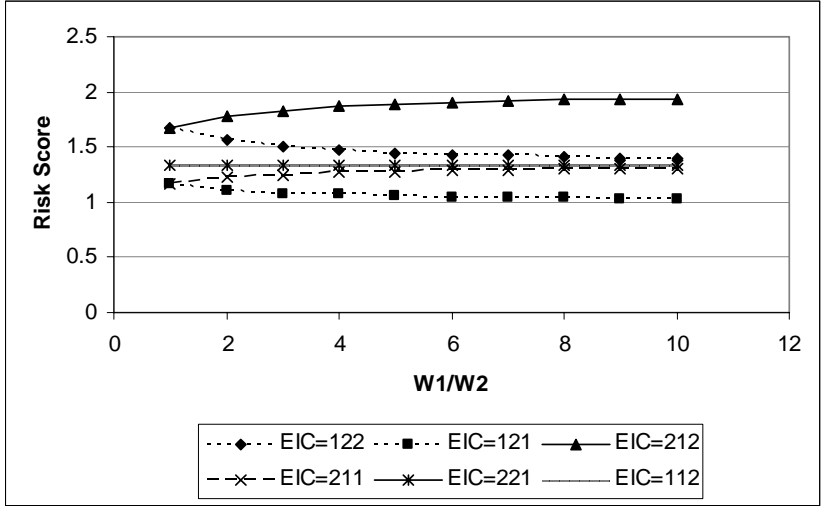

Figure 3. Variation of risk score with relative weight of environment/ inspectability 
The notation EIC = 122 represent the group of bridges which belongs to Mild Environment $(\mathrm{E}=1)$, Hard Inspectability (I = 2) and High Consequence $(C=2)$. Altogether 8 groups can be obtained by changing this combination. However, the group with mild environment, easy inspectability and low consequence $(E I C=111)$ and the group with severe environment, hard inspectability and high consequence $(E I C=222)$ are not plotted in the graph as their scores always remain as 1 and 2 respectively. From, this graph it can be seen that the variation in the risk scores with the relative weights is small, particularly with weight factors higher than 3 . Therefore, a maximum relative weight of 3 between the environment and inspectability factor can be used.

A new method for incorporating the weight factor is proposed and the sensitivity tested again. In this method the worse case is assigned a weight of 3 . That is, if the environment is severe, then use a relative weight 3 for the environment and if the inspectability is hard, assign inspectability a weight of 3. In the cases where both environment and inspectability are in worse or better conditions, the weight factors have no effect. The scores obtained using this approach and the scores obtained without using weight factors are given in Table 1.

It can be seen from Table 1, that the difference between the two methods is small with a maximum percentage difference of 9.6\%. Based on the results of the sensitivity studies and bearing in mind that the scoring system is only a qualitative measure of the relative risk among the sub groups of bridges it was decided to ignore the weighting factors from the scoring system. Therefore the relative risk score ' $R$ ' is calculated using Equation 2.

$\mathrm{R}=\left(\mathrm{S}_{\mathrm{E}}+\mathrm{S}_{\mathrm{I}}\right) \times \mathrm{S}_{\mathrm{C}}$

Table 1. Differences in scores with and without weight factors

\begin{tabular}{|c|c|c|c|}
\hline \multirow[b]{2}{*}{ Condition } & \multicolumn{2}{|c|}{ Score } & \multirow{2}{*}{$\begin{array}{c}\text { Percentage } \\
\text { Difference } \\
\text { (\%) }\end{array}$} \\
\hline & $\begin{array}{c}\text { without } \\
\text { weight factor }\end{array}$ & $\begin{array}{c}\text { with weight } \\
\text { factor }\end{array}$ & \\
\hline $\mathrm{EIC}=111$ & 1.00 & 1.00 & 0 \\
\hline $\mathrm{EIC}=112$ & 1.33 & 1.33 & 0 \\
\hline $\mathrm{EIC}=121$ & 1.17 & 1.25 & 6.8 \\
\hline $\mathrm{EIC}=122$ & 1.67 & 1.83 & 9.6 \\
\hline $\mathrm{EIC}=211$ & 1.17 & 1.25 & 6.8 \\
\hline $\mathrm{EIC}=212$ & 1.67 & 1.83 & 9.6 \\
\hline $\mathrm{EIC}=221$ & 1.33 & 1.33 & 0 \\
\hline $\mathrm{EIC}=222$ & 2.00 & 2.00 & 0 \\
\hline
\end{tabular}

These values are normalized to vary between 1 and 2 by linear interpolation. This adjustment was considered desirable so that 1 and 2 always represent the best and worse cases respectively. The risk ranking system is provided in Table 2.
Table 2. Risk ranking system

\begin{tabular}{llll}
\hline Environment & Consequence & Inspectability & Risk Score \\
\hline Mild & Low & Easy & 1.00 \\
Mild & Low & Hard & 1.17 \\
Mild & High & Easy & 1.33 \\
Mild & High & Hard & 1.67 \\
Severe & Low & Easy & 1.17 \\
Severe & Low & Hard & 1.33 \\
Severe & High & Easy & 1.67 \\
Severe & High & Hard & 2.00 \\
\hline
\end{tabular}

\section{APPLICATION OF RISK RANKING STRATEGY TO NETWORK RAIL BRIDGE STOCK}

The proposed risk ranking strategy is demonstrated through its application on the UK Network Rail bridge stock that comprises over 40,000 bridges. This includes bridges carrying railway traffic (under line bridges) and bridges carrying roads which pass over the railway lines (over line bridges). Initially the main groups are identified based on the 'type' attribute and then the criteria to rank the bridges within those main groups are developed.

\subsection{Identification of main group}

Analyzing Network Rail bridge stock it was observed that about half of the network bridges are masonry arch bridges, either brick or stone, and almost all of them are over 100 years old. Metallic bridges are about $40 \%$ of the stock and spread over three age ranges $<50,50-100 \&>100$ years. Concrete has been used in the remaining bridges and were constructed mostly within the last 50 years (Bell,2004). It was also observed that metallic bridges can be classified in to three types, namely cast iron riveted steel and welded steel. Therefore, based on the forms and age of the bridges in the network following six main groups are proposed; stone arch bridges, masonry arch bridges, cast iron bridges, riveted steel bridges, welded steel bridges and concrete bridges.

\subsection{Classification criteria for environment}

The environment of a bridge will be considered as severe if two or more of the following factors are severe/heavy.

\subsubsection{Loading}

Loading is one of the external factors that affect deterioration due to fatigue and/or ring separation. A qualitative classification of loads has been used that is based on the type of traffic. Railway routes are classified by the Network Rail into the following categories; primary, LSE (London South Eastern), main secondary, secondary, rural and freight. Among them, primary, LSE and freight routes are 
considered to carry heavy traffic and the bridges in these routes are considered as heavily loaded bridges. Over line bridges serving motorways and trunk roads can also be considered as heavily loaded bridges.

\subsubsection{Climate of the area}

Frost and rain are the two most common causes for the deterioration of mortar and brick. Therefore, the bridges can be classified into two groups in terms of frost and rainfall. Based on the annual average rain fall and frost level records from the Met office website(http://www.metoffice.gov.uk/climate/uk/average s/index.html) the climate of the UK can be classified into two regions; severe and mild. In this procedure, regions having annual rainfall under $1125 \mathrm{~mm}$ or regions with air frost less than 55 days are considered as mild since these are the national average values.

\subsubsection{Location of bridge}

Susceptibility of masonry bridges due to its location is relatively low when compared to concrete or metallic bridges. Concrete or metallic bridges are prone to corrosion attack if they are exposed to salty environment. However, masonry bridges in highly polluted industrial areas also can be taken as severe in terms of the location since the mortar in bridges is vulnerable to chemical attacks.

\subsection{Classification criteria for consequence}

Factors identified as having influence on the consequence (see Figure. 1) are categorized into two groups, namely 'high' and 'low'. If two or more of the factors are classified as high, then the consequence factor is considered as 'high'.

\subsubsection{Railway traffic flow}

Railway traffic flow can be considered as an indirect indicator of the possible consequences of an underline bridge failure such as human life loss/or injuries, loss of income, etc. It is assumed that the underline bridges in primary and LSE lines have high traffic flow rate.

\subsubsection{Road traffic flow}

Over line bridges carry road traffic. Therefore, their failure consequence can be estimated in a similar manner to the underline bridges. Motorways and trunk roads can be assumed to have higher traffic flow rate. In addition, bridges on traffic sensitive roads (as designated by the Street works act http://www.opsi.gov.uk/si/si2007/20071951.htm) can be considered as of high consequence.

\subsubsection{Cost and/or duration of remedial actions}

Cost and duration of remedial actions are difficult to estimate as they will be more bridge specific and will depend on the type of damage. However, it is assumed that the bridges with multi or long spans may encounter high cost and/or duration. This includes an implicit assumption that all spans of a multi span bridge perform in a similar manner.

\subsection{Classification criteria for inspectability}

The inspectability can be affected in two ways. Firstly, inspections will not be able to reveal condition of hidden details of the bridge and the other factor is the difficulties in access to the bridge due to its location. This information is obtained from the past inspection reports. Normally, the examiners make a note in the examination report whether or not they were able to examine all major parts of the bridge.

\subsection{Case studies on selected bridges}

In this section a random selection of masonry bridges from Network Rail are ranked according to the above guidelines to demonstrate the procedure. The railway traffic type and SCMI details are obtained electronically from the Network Rail database. The locations of bridges are obtained from past inspection records and they are compared with the rain fall and frost maps to identify the relevant climatic condition. The roads adjacent to the bridges are identified using www.streetmap.co.uk and used to establish the consequence attribute. The required information about the inspectability is obtained from the past inspection reports.

As an example, asset Reference ANG030 is a masonry underline bridge in a primary line near to Harlesden area. From the met office records the rain fall and frost are identified as low. There are no noticeable heavily pollutant industries near the bridge. Therefore, the environment is taken as 'mild'. Its land ranger is TQ210839. From streetmap.com the road adjacent to this is A407 which is not a critical road. However, since this bridge is a multi span bridge and it is on a primary line its consequence is 'high'. Past inspection record for this bridge indicates no inspection difficulties or hidden details of the structure hence inspectability is assigned as 'easy'. Therefore, this bridge has mild environment, high consequence and easy inspectability, hence according to Table 2, a risk score of 1.33 is assigned to this bridge. Table 3 presents a list of sample structures ranked according to their relative risk.

These risk scores can be used to establish the inspection strategy within a main group of bridges. Since they are indications of the relative risk levels of the bridges, selecting inspection intervals proportional to these scores would ensure same risk level is maintained. For instance, if the bridges with the score of 1.00 are inspected at 6 year intervals, then the bridges with score of 1.33 must be inspected every $4 \frac{1}{2}$ years to maintain the consistent risk levels within bridges. A method to identify the inspection 
intervals for bridges with score 1.00 is currently being developed by the authors.

Table 3. Bridge groups and SCMI scores

\begin{tabular}{lllll}
\hline Asset Ref & E & C & I & $\begin{array}{l}\text { Risk } \\
\text { Score }\end{array}$ \\
\hline ANG034 & Mild & Low & Easy & 1.00 \\
ANG006 & Mild & Low & Easy & 1.00 \\
NW006 & Mild & Low & Easy & 1.00 \\
GW006 & Mild & Low & Easy & 1.00 \\
ANG031 & Mild & Low & Easy & 1.00 \\
LNW-MID002 & Mild & Low & Easy & 1.00 \\
STH030 & Mild & Low & Easy & 1.00 \\
ANG033 & Mild & Low & Hard & 1.17 \\
LNE030 & Mild & Low & Hard & 1.17 \\
ANG007 & Mild & Low & Hard & 1.17 \\
SCO104 & Severe & Low & Hard & 1.17 \\
LNE-MID001 & Mild & High & Hard & 1.33 \\
LNE008 & Mild & High & Hard & 1.33 \\
ANG030 & Mild & High & Easy & 1.33 \\
LNE-MID106 & Mild & High & Hard & 1.33 \\
SCO103 & Severe & Low & Hard & 1.33 \\
LNW-MID025 & Severe & Low & Easy & 1.33 \\
GW101 & Severe & High & Easy & 1.67 \\
\hline
\end{tabular}

\section{CONCLUSION}

The development of a risk ranking strategy is presented in this paper, which can serve as a screening process to rank the bridges in a network according to their relative risk levels. Main factors that may affect risk in relation to safety and serviceability of bridges are identified and linked to various bridge attributes. These attributes can be used to divide a network of bridges into groups according to their relative risks. A scoring system is developed to rank the groups in terms of their relative risk. Sensitivity analysis yielded the effects of different weights of the attributes on the relative risk scores.

The proposed method is demonstrated through its application on the UK railway network bridge stock. Six main groups are proposed based on the type attribute. Eight subgroups within each main group are proposed using the environment, consequence and inspectability attributes. Each of the attributes is classified into two categories based on the severity and relative risk score for each group is established.

The classification system and the use of attributes in deriving relative risk is demonstrated on a sample of bridges from the network.

The risk ranking methodology will help the bridge owners to identify critical bridges in their network in a systematic and rational manner. The risk scores along with the deterioration profiles for each bridge type can be used to establish inspection / intervention intervals for each group. This will ensure that a consistent risk level is maintained over the network. A process for the risk based inspection planning is being developed and will be published in the near future.

\section{Acknowledgements}

The authors express their sincere gratitude to Network Rail for sponsoring this project and Mouchel for providing data. We also thank the steering committee members from various bridge related organizations who attended regular meetings for their valuable comments. We extend our sincere thanks to Brian Bell from Network Rail for all his contributions to this project.

\section{REFERENCES:}

Bell, B. 2004. European railway bridge demography, Deliverable D 1.2, Technical report, UK: Sustainable bridges, Sixth framework programme, Sustainable development global change \& ecosystems.

Daly, A.F. 1999. Modelling of deterioration in bridges, Deliverable D11, European Commission DG VII-4th Framework Programme. Bridge Management in Europe. Available from: http://www.trl.co.uk/brime/d11.pdf

Faber, M.H. Kroon, I.B. \& Sorensen, J.D. 1996. Sensitivities in structural maintenance planning. Reliability engineering and system safety $51:$ 317-329.

Frangopol, D.M. Lin, K.Y. \& Estes, A.C. 1997. Life cycle cost design of deteriorating structures. Journal of structural engineering 123(10): 1390-1401.

Liu, M. \& Frangopol, D.M. 2005. Multi-objective maintenance planning optimization for deteriorating bridges considering condition, safety, and life-cycle cost. Journal of structural engineering 131(5): 833-842.

Mckibbins, L.D. Melbourne, C. Sawar, N. \& Gaillard, C.S. 2006. Masonry arch bridges: Condition appraisal and remedial treatment-CIRIA C656. London: CIRIA.

Melbourne, C. \& Gilbert, M. 1995. The behaviour of multiring brickwork arch bridges. Structural Engineer 73(3): 39-47.

Onoufriou, T. 1999. Reliability based inspection planning for offshore structures, Journal of Marine Structures 12(7-8): 521-539.

Page, J. 1993. Masonry arch bridges, London: TRL. HMSO.

SACP, pers. comm. 2005. SACP Feasibilities, London: Mouchel Parkman

Sommer, A.M. Nowak, A.S. \& Thoft-Christension, P. 1993. Probability based bridge inspection strategy, Journal of structural engineering 119(12): 3521-3535.

Stupart, A.W. 1989. A survey of literature relating to frost damage in bricks. Masonry international 3(2): 42-50.

Yang, J.N. \& Trapp, W.J. 1974, Reliability analysis of aircraft structures under random loading and periodic inspection, AIAA Journal 12(12): 1623-1630.

Www.metoffice.gov.uk

http://www.opsi.gov.uk/si/si2007/20071951.htm

www.streetmap.co.uk 